\title{
Quantitative analysis of the flavonoid content of blueberry extracts by liquid chromatography - high resolution - mass spectrometry
}

Blueberries are a rich source of polyphenols, namely anthocyanins ${ }^{(1)}$. Polyphenols may be one of the key components playing a contributive role in the protective effect of fruits and vegetables against non-communicable diseases. However, the health impact of any compound is firstly dependent on i) its concentration in the food and ii) its bioavailability. The concentration of bioactives in food can be impacted on by a range of factors such as, variety, seasonal and climate changes, processing etc. Here, we carried out a quantitative analysis of phenolic compounds present in a range of blueberry varieties (Collins, Blue Crop, Spartan, Berkley and Blue Ray) collected over 3 consecutive years (2010, 2011 and 2012). Samples were analysed in triplicate using ultra performance liquid chromatography - photodiode array high resolution - mass spectrometry (HPLC-PDA-HR-MS, Thermo Accella UPLC), injected onto a $100 \times 2 \cdot 1 \mathrm{~mm} \mathrm{C}_{18} \mathrm{Hypersil} \mathrm{Gold}$ Column (Thermo Fisher) and eluted with a 5-40\% gradient of $1 \%$ formic acid and methanol at $400 \mu \mathrm{L} / \mathrm{min}$ over 0-20 minutes. All samples were run in positive ion mode using high-resolution $(50,000)$ exact mass for compound identification with a scan range of $250-1500$ ати. A total of 28 compounds were detected and quantified (Fig. 1). The main components were anthocyanins with a minimum concentration of $8.9 \pm 2.0 \mathrm{mg} / \mathrm{g}$ and maximum concentration of $19.8 \pm 2.1 \mathrm{mg} / \mathrm{g}$, corresponding to $55 \%-64 \%$ of total polyphenols, followed by flavonols with quercetin-3-galactoside the major flavonol in all the blueberries varieties. A trend was detected when comparing the three crop years, with 2011 as a better growing year in terms of polyphenolic content, with the exception of the Berkley variety.
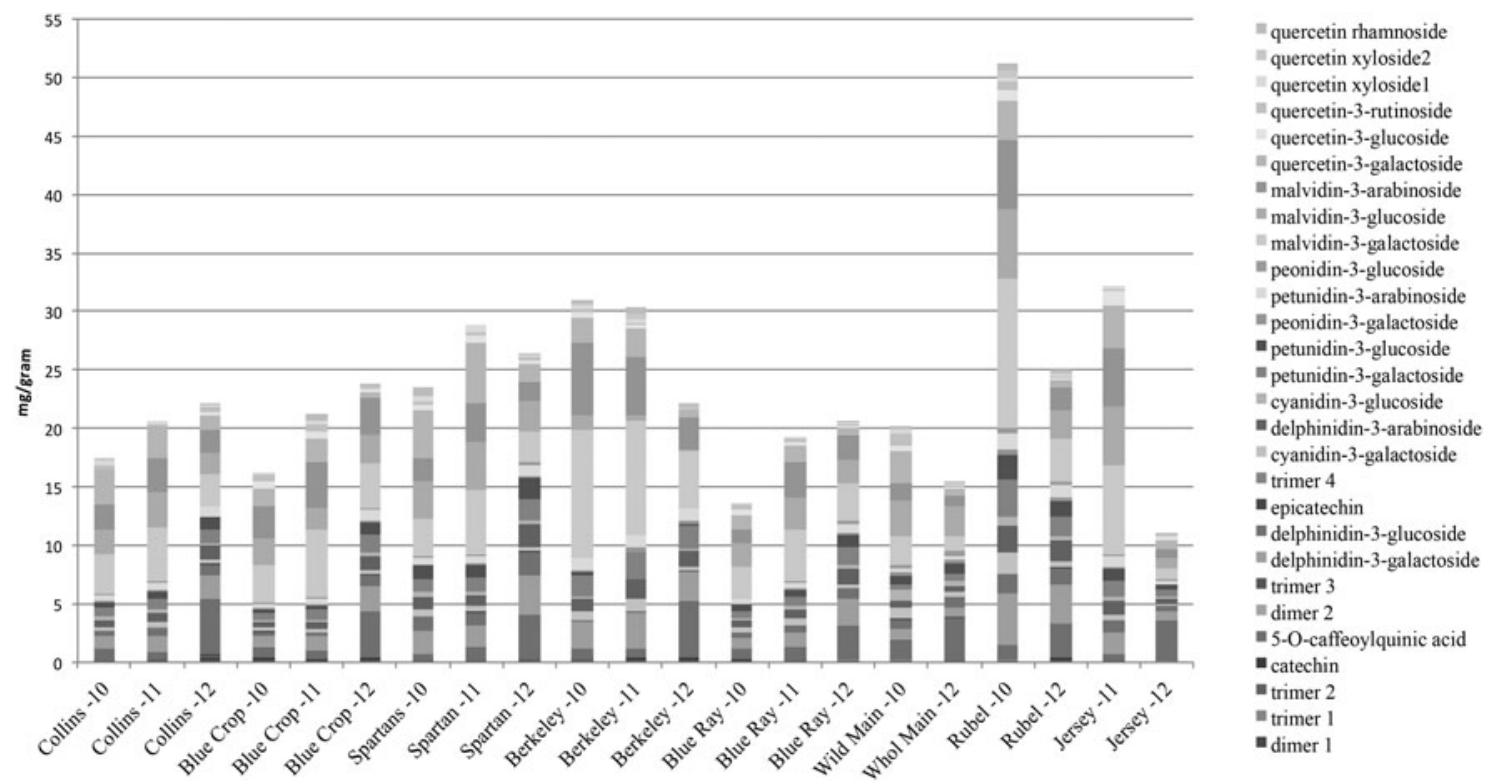

Fig. 1. Mean total polyphenol content of blueberries analysed in triplicate with 3 year data; contribution from individual compounds.

It can be demonstrated that there is a variety in phenlolic content across the blueberry varieties as well as the year of crop. Although 2012 was reported as a year of crop failure, the polyphenolic content of the blueberries was not adversely affected. This could be interpreted in terms of these compounds being produced in times of attack or stress as a defense mechanism.

1. Mullen W, Marks SC, Crozier A, (2007). Evaluation of phenolic compounds in commercial fruit juices and fruit drinks. Journal of Agricultural and Food Chemistry. 55(8), 3148-57. 